\title{
Nanorod Manganese Oxide as an Efficient Heterogeneous Catalyst for Hydration of Nitriles into Amides
}

Hai Wang,,$^{\dagger}$ Yeqing Wang, ${ }^{*}+\dot{+}$ Hua Xu,,$^{+}$Hang Zhou, ${ }^{\dagger}$ Liang Wang, ${ }^{\dagger}$ Xiangju Meng, ${ }^{+}$

Feng-Shou Xiao ${ }^{*,+, t}$

$\dagger$ Key Lab of Biomass Chemical Engineering of Ministry of Education, College of Chemical and Biological Engineering, Zhejiang University, Hangzhou 310027, China.

$\$$ Department of Chemistry, Zhejiang University, Hangzhou 310028, China.

*Corresponding author

E-mail: wangyeqing@zju.edu.cn; fsxiao@zju.edu.cn 


\section{CONTENT}

Scheme S1. Hydration of benzonitrile with $\mathrm{D}_{2} \mathrm{O}$ into D-labeled amide product over $\mathrm{NR}-\mathrm{MnO}_{2}$ catalyst.

Figure S1. XRD pattern of $\mathrm{NR}-\mathrm{MnO}_{2}$.

Figure S2. STEM image, elemental map, and EDX spectrum of $\mathrm{NR}-\mathrm{MnO}_{2}$.

Figure S3. GC curve of the liquor after benzonitrile hydration over $\mathrm{NR}-\mathrm{MnO}_{2}$ catalyst.

Figure S4. XRD patterns.

Figure S5. Benzonitrile hydration at different temperatures.

Figure S6. Dependences of benzamide yields and selectivities on time in the benzonitrile hydration over $\mathrm{NR}-\mathrm{MnO}_{2}$ catalyst.

Figure S7. Photographs of the reaction mixture.

Figure S8. Recycling test of $\mathrm{NR}-\mathrm{MnO}_{2}$ catalyst.

Figure S9. XRD pattern of the $\mathrm{NR}-\mathrm{MnO}_{2}$ catalyst after the $5^{\text {th }}$ run.

Figure S10. TEM image and the diameter distribution of the $\mathrm{NR}-\mathrm{MnO}_{2}$ catalyst after $5^{\text {th }}$ run.

Figure S11. Average reaction rate $\left(r_{0}\right)$ and benzamide selectivity in the hydration of benzonitrile over $\mathrm{NR}-\mathrm{MnO}_{2}$ catalyst using $\mathrm{H}_{2} \mathrm{O}$ and $\mathrm{D}_{2} \mathrm{O}$.

Figure S12. GC-MS spectra of the D-labeling experiment in benzonitrile hydration over NR- $\mathrm{MnO}_{2}$ catalyst.

Figure S13. The yields and selectivities of benzamide in the benzonitrile hydration with $\mathrm{H}_{2} \mathrm{O}$ and $\mathrm{D}_{2} \mathrm{O}$.

Figure S14. Hammett plots for the hydration of different $p$-substituted benzonitriles 
over the NR-MnO 2 catalyst.

Table S1. Catalytic performance of various homogeneous catalysts in hydation of benzontrile.

Table S2. Catalytic data of various catalysts in hydation of benzontrile.

Table S3. Catalytic data of $\mathrm{NR}-\mathrm{MnO}_{2}$ in hydation of benzontrile at different temperatures.

Table S4. Catalytic data characterizing the NR-MnO 2 catalyst in hydation of benzontrile at different time.

Table S5. Catalytic data in hydation of various ntriles over NR-MnO 2 . 


\section{EXPERIMENTAL SECTION}

\section{Catalyst Characterization}

Powder X-ray diffraction patterns (XRD) were obtained on a Rigaku D/MAX 2550 diffractometer with $\mathrm{Cu} \mathrm{K} \alpha$ radiation $(\lambda=0.1542 \mathrm{~nm})$. Metal leaching was determined by inductively coupled plasma optical emission spectrometry analysis (ICP-OES, Perkin-Elmer 3300DV). Transmission electron microscopy (TEM), scanning transmission electron microscopy (STEM) images and energy dispersive spectroscopy were carried out on a Cs-corrected JEM-ARM300F electron microscopy (JEOL, Japan) with an acceleration voltage of $300 \mathrm{kV}$. Typically, the samples were ultrasonically dispersed in ethanol and then a drop of the mixture was added onto a holey $\mathrm{C} / \mathrm{Cu}$ grid for TEM characterization.

\section{Catalytic tests}

Recycling test and hot filtration experiment. In the recycling tests, the catalysts were separated from the reaction liquid by centrifugation after each reaction run, and washed with ethanol and deionized water for several times, dried at $150{ }^{\circ} \mathrm{C}$ overnight, and used again in the next run. Hot filtration was performed to evaluate the catalyst leaching in the reaction liquor. ${ }^{1}$ After hydration of benzonitrile for $0.5 \mathrm{~h}$ at $75{ }^{\circ} \mathrm{C}$ over $\mathrm{NR}-\mathrm{MnO}_{2}$ ( $0.4 \mathrm{mmol}$ of benzonitrile, $100 \mathrm{mg}$ of NR-MnO $2,4 \mathrm{~mL}$ of $t$-amyl alcohol solvent, 100 $\mu \mathrm{L}$ of $\left.\mathrm{H}_{2} \mathrm{O}\right)$, the hot reaction mixture was immediately filtrated by a filter $(0.22 \mathrm{um})$ and used in the next run without additional catalyst.

In the catalytic tests, the nitriles conversion, products yield and selectivity in the hydration of different nitriles were determined according to the following equations: 


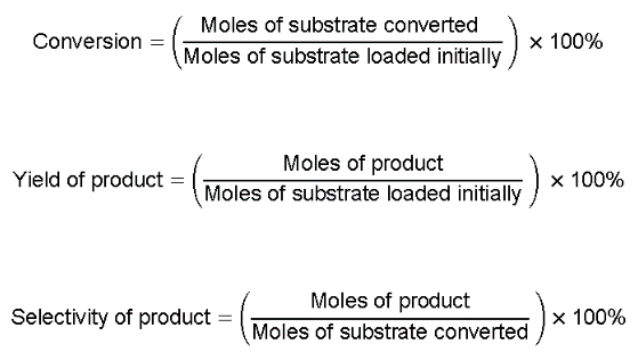

In kinetic study, the nitrile conversions were controlled to be lower than $20 \%$, and the average reaction rates $\left(r_{0}\right)$ were calculated from the moles of substrate converted per gram of catalyst per hour $\left(\mathrm{mol} \mathrm{g}_{\mathrm{cat}}{ }^{-1} \mathrm{~h}^{-1}\right)$.

Carbon balance in the hydration of nitriles. The carbon balance was calculated on the basis of the number of carbon atoms in the reactor before and after the reactions. The carbon balance values are calculated according to the following equation:

$$
\mathrm{C} \%=\frac{M_{f} * 7+M_{1} * n_{1}+M_{2} * n_{2}+M_{3} * n_{3}+\cdots+M_{x} * n_{x}}{M_{e} * 7} * 100 \%
$$

Where $\mathrm{C} \%$ is the value of carbon balance values, $\mathrm{M}_{\mathrm{f}}$ is the final moles of benzonitrile in the reactor after reaction; 7 is the number of carbon atoms in a benzonitrile molecule; $\mathrm{M}_{1}\left(\mathrm{M}_{2}, \mathrm{M}_{3} \ldots \mathrm{M}_{\mathrm{x}}\right)$ is the moles of liquid product 1 (product $\left.2,3 \ldots \ldots \mathrm{x}\right)$ in the reactor after reaction; $\mathrm{n}_{1}\left(\mathrm{n}_{2}, \mathrm{n}_{3} \ldots . . \mathrm{n}_{\mathrm{x}}\right)$ is the number of carbon atoms in a single molecule of product 1 (product 2, 3.....x); $\mathrm{M}_{\mathrm{e}}$ is the mole of benzonitrile in the feed. 


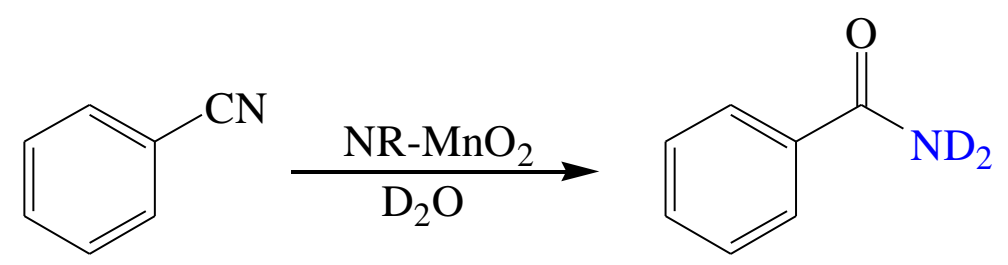

Scheme S1. Hydration of benzonitrile with $\mathrm{D}_{2} \mathrm{O}$ into D-labeled amide product over $\mathrm{NR}-\mathrm{MnO}_{2}$ catalyst. 


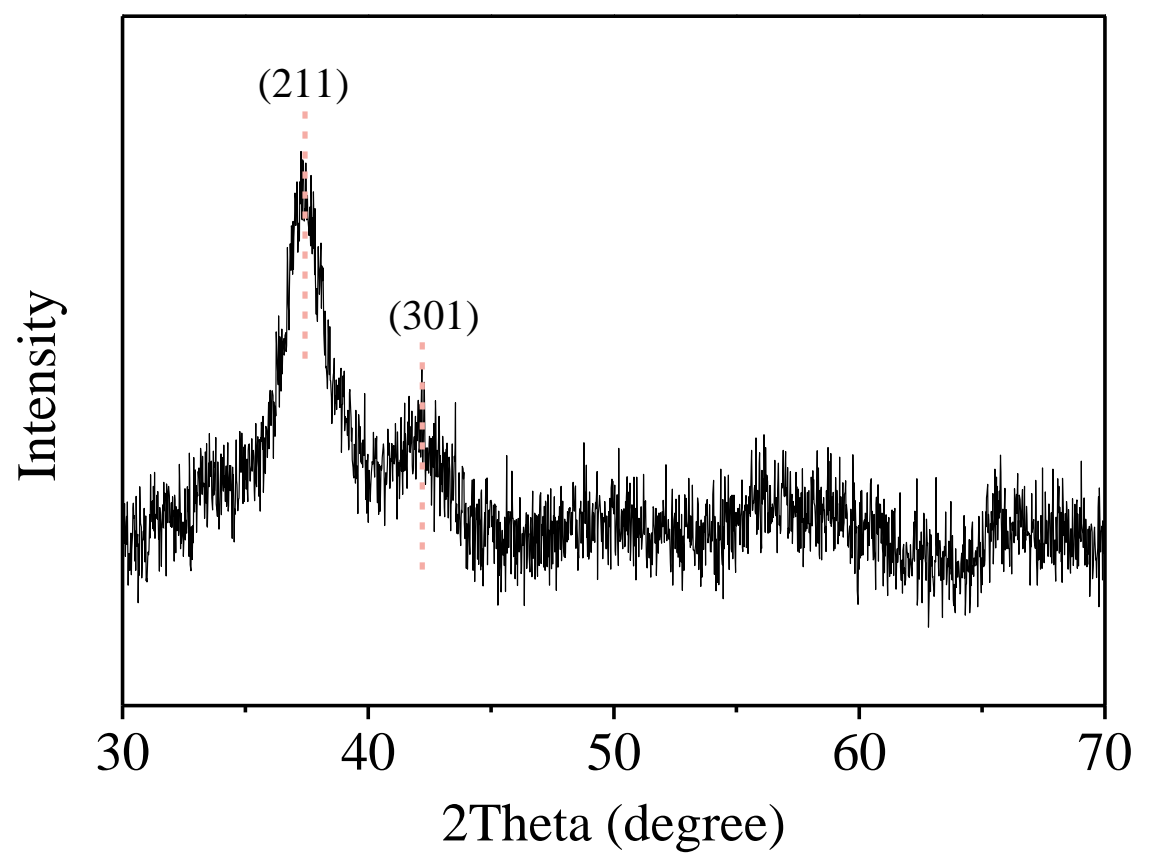

Figure S1. XRD pattern of NR-MnO 2 . 


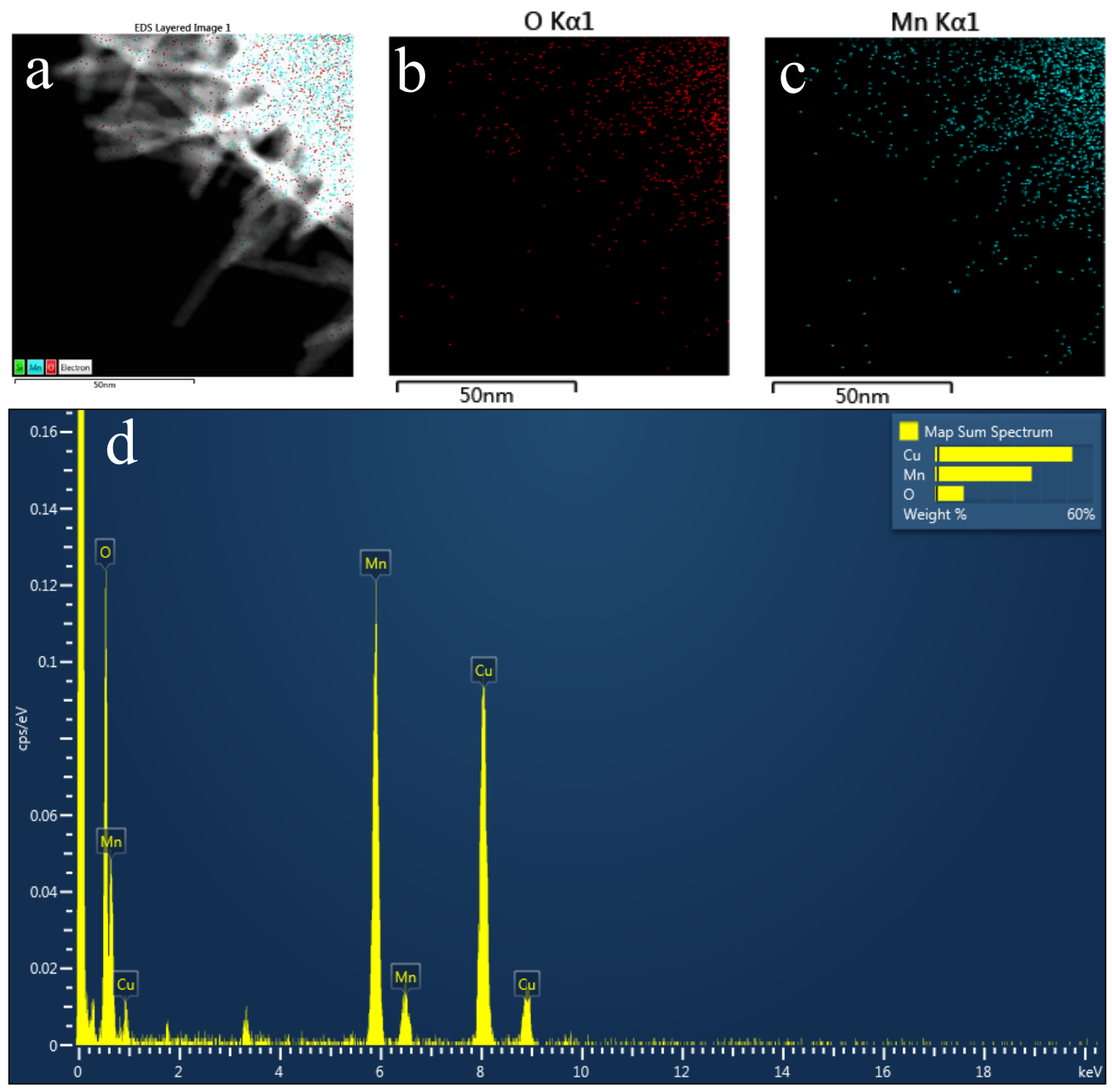

Figure S2. (a) STEM image the corresponding (b) O and (c) Mn elemental map, and (d) EDX spectrum of NR-MnO 2 sample. 


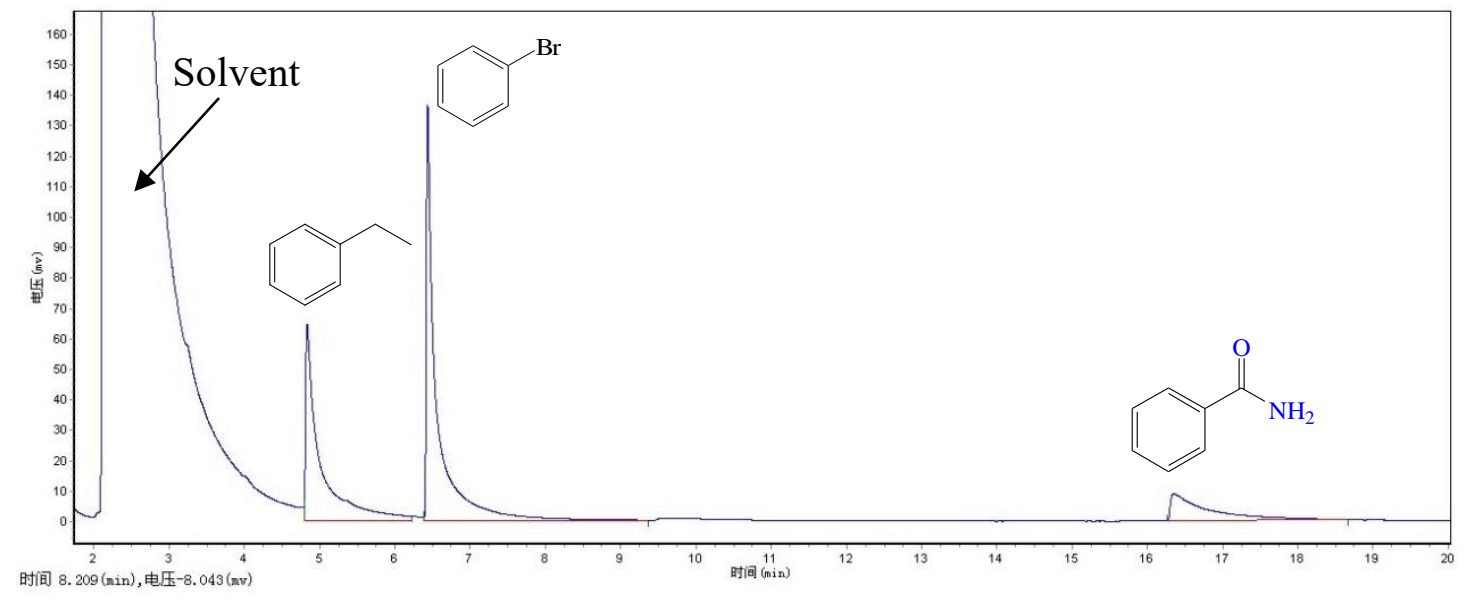

Figure S3. GC curve of the liquor after benzonitrile hydration over $\mathrm{NR}-\mathrm{MnO}_{2}$

catalyst. Ehtlybenzene and bromobenzene were used as internal standards. 

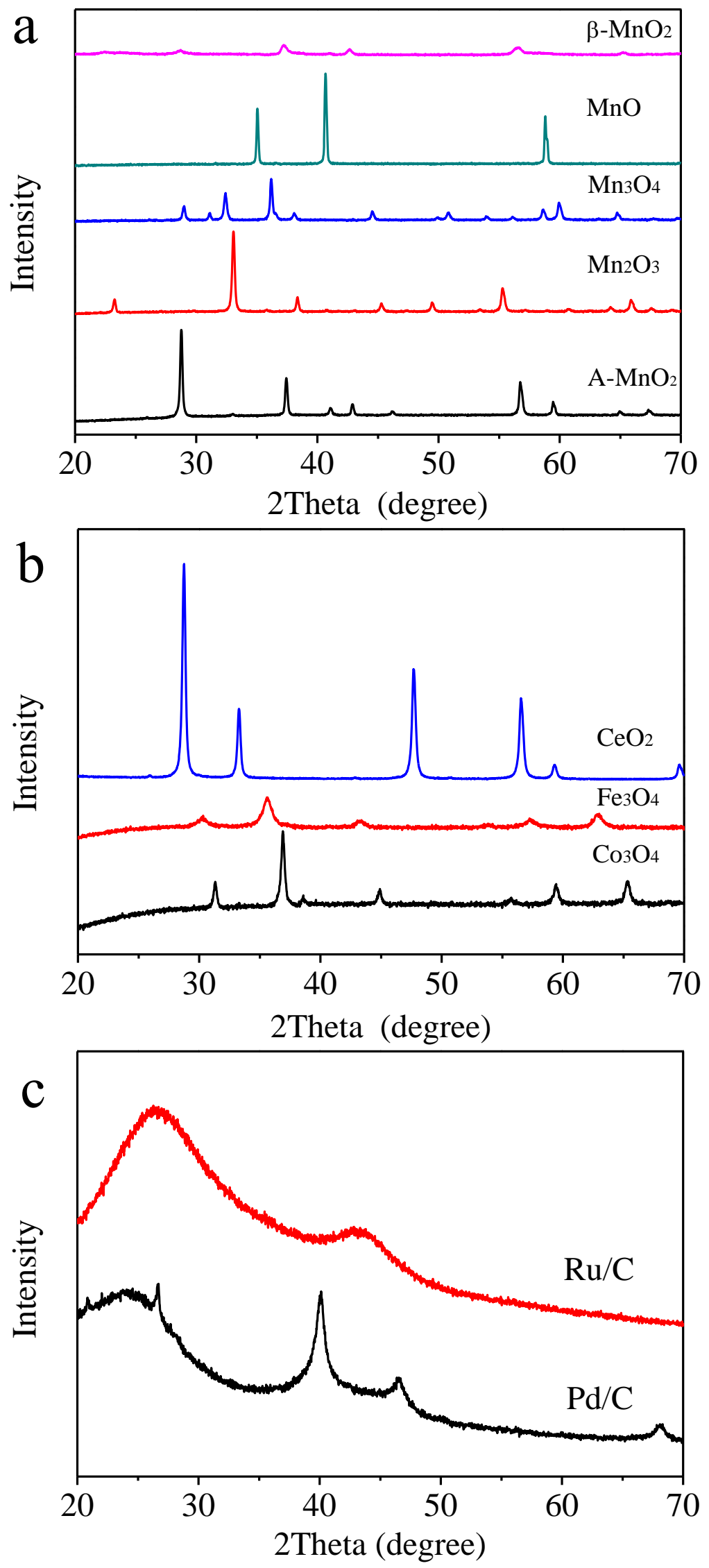

Figure S4. XRD patterns of various samples including (a) manganese oxides, (b) other oxides, and (c) noble-metal based catalysts. 


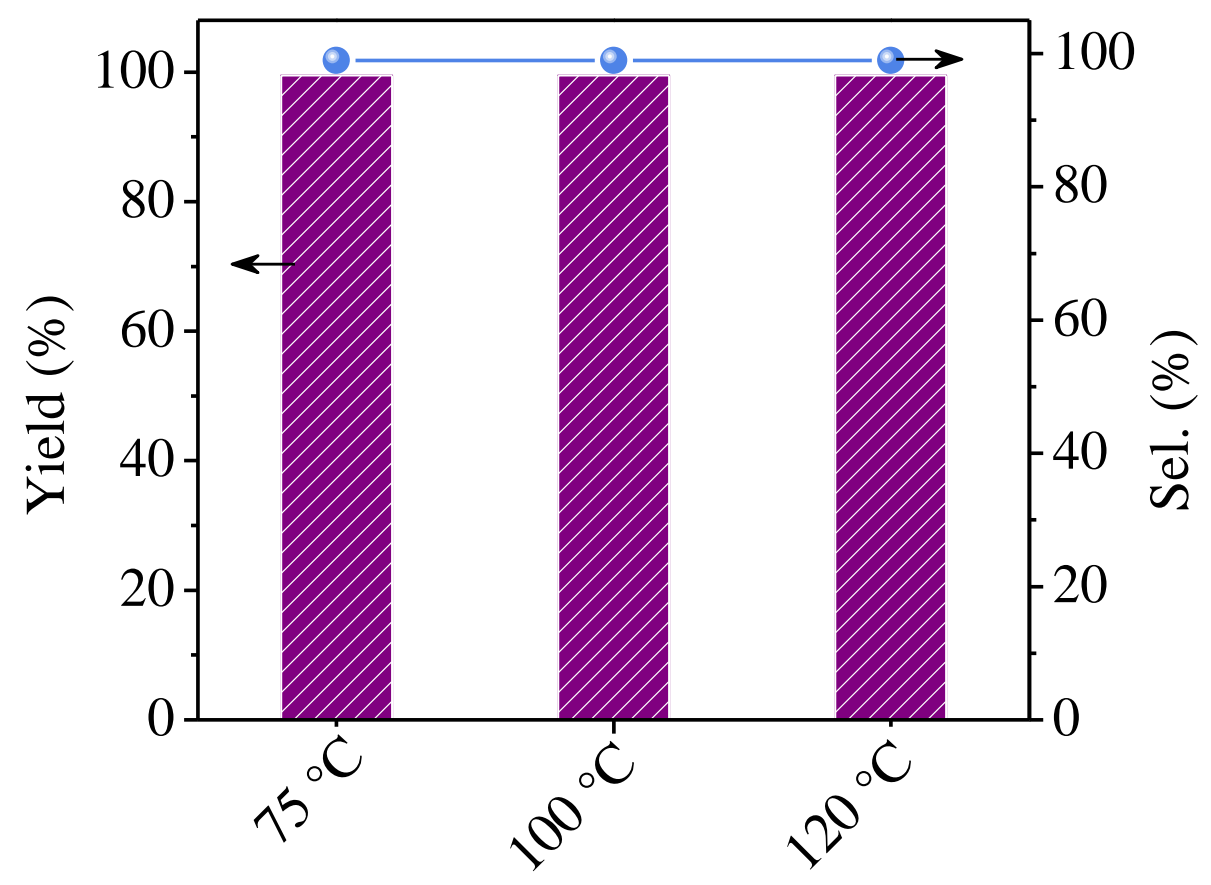

Figure S5. Data characterizing the performances of $\mathrm{NR}-\mathrm{MnO}_{2}$ catalyst in benzonitrile hydration at different temperatures. Reaction conditions: $0.4 \mathrm{mmol}$ of substrate, $4 \mathrm{~mL}$ of $t$-amyl alcohol, $100 \mu \mathrm{L}$ of $\mathrm{H}_{2} \mathrm{O}, 100 \mathrm{mg}$ of catalyst, $2 \mathrm{~h}$. More data are presented in Table S3. 


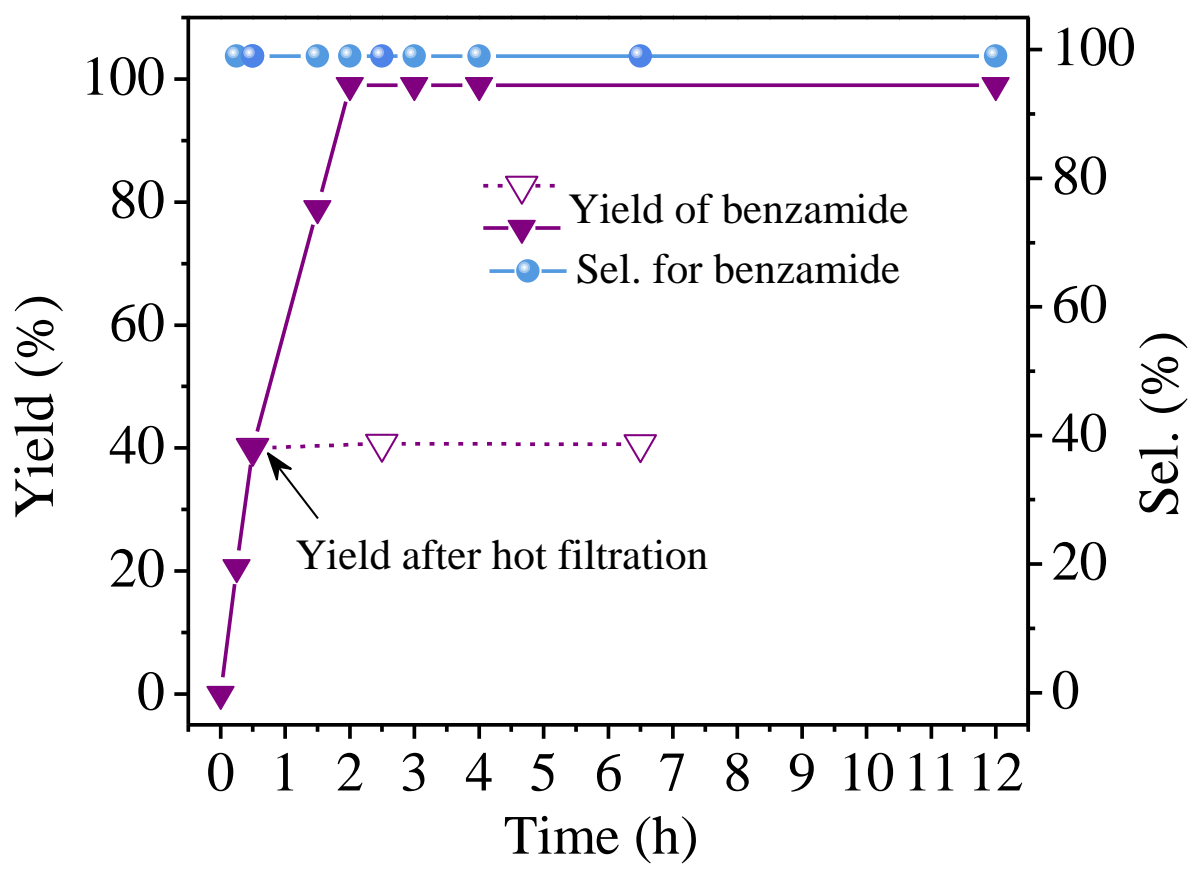

Figure S6. Dependences of benzamide yields and selectivities on time in the benzonitrile hydration over $\mathrm{NR}-\mathrm{MnO}_{2}$ catalyst, the close and open purple lines are obtained via two separate experiments. Reaction conditions: $0.4 \mathrm{mmol}$ of substrate, 4 $\mathrm{mL}$ of $t$-amyl alcohol, $100 \mu \mathrm{L}$ of $\mathrm{H}_{2} \mathrm{O}, 100 \mathrm{mg}$ of catalyst, $75{ }^{\circ} \mathrm{C}$. More data are presented in Table S4. 

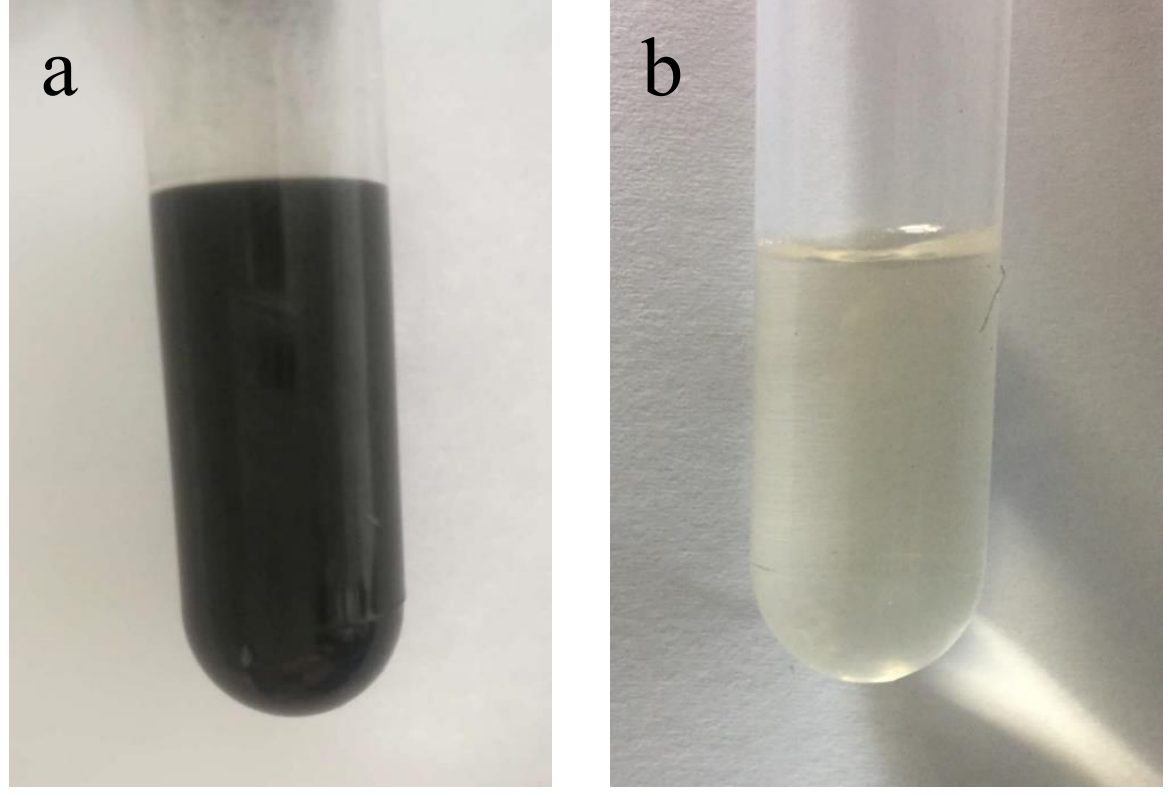

Figure S7. Photographs of the (a) reaction mixture during reaction and (b) liquor after removing $\mathrm{NR}-\mathrm{MnO}_{2}$ catalyst by hot filtration at $75{ }^{\circ} \mathrm{C}$. 


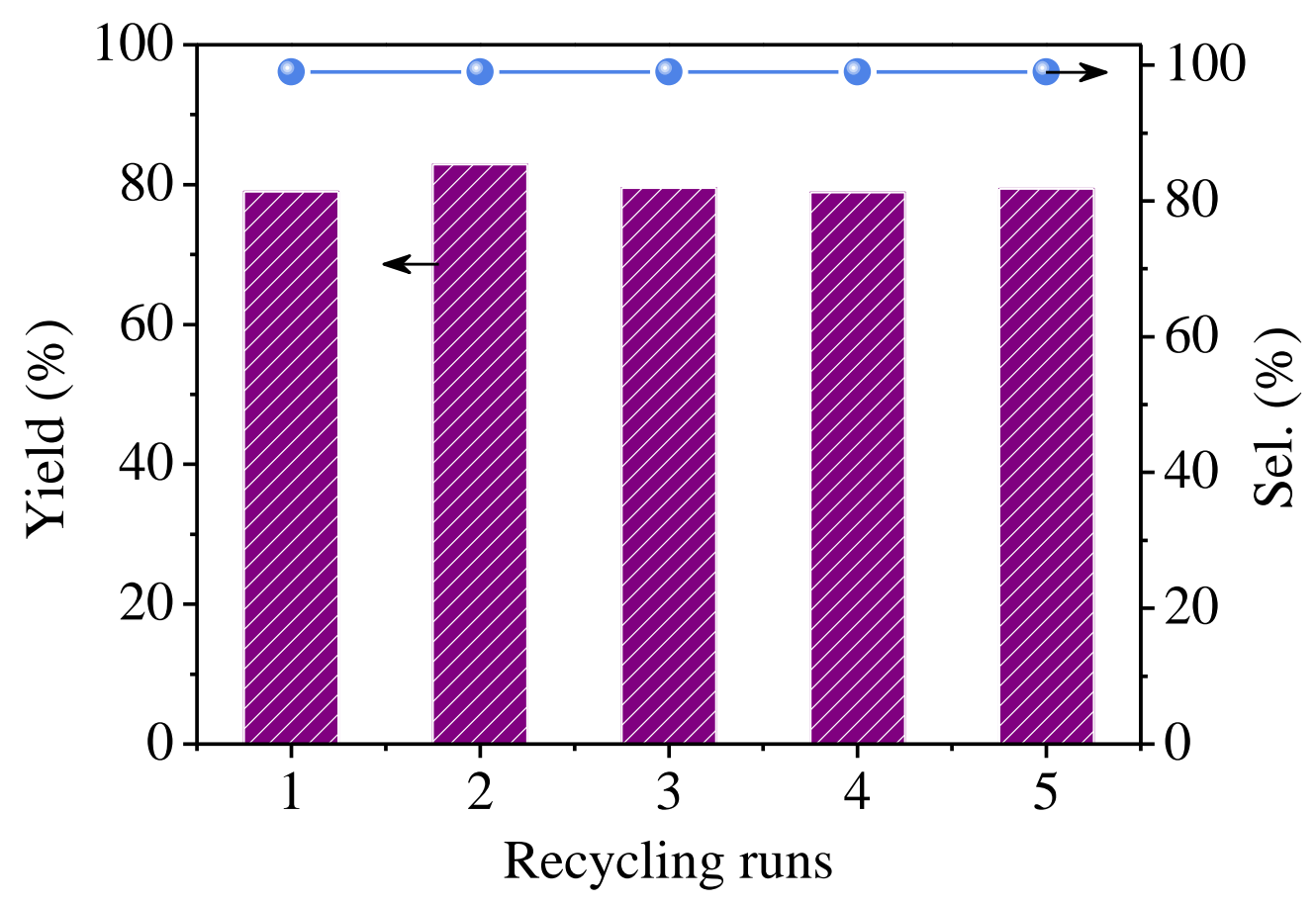

Figure S8. Data characterizing the performances of $\mathrm{NR}-\mathrm{MnO}_{2}$ catalyst in the recycling test. Reaction conditions: $1.0 \mathrm{mmol}$ of substrate, $10 \mathrm{~mL}$ of $t$-amyl alcohol, $250 \mu \mathrm{L}$ of $\mathrm{H}_{2} \mathrm{O}, 250 \mathrm{mg}$ of catalyst, $75^{\circ} \mathrm{C}, 1.5 \mathrm{~h}$. Yield: yield of benzamide, Sel.: selectivity for benzamide. 


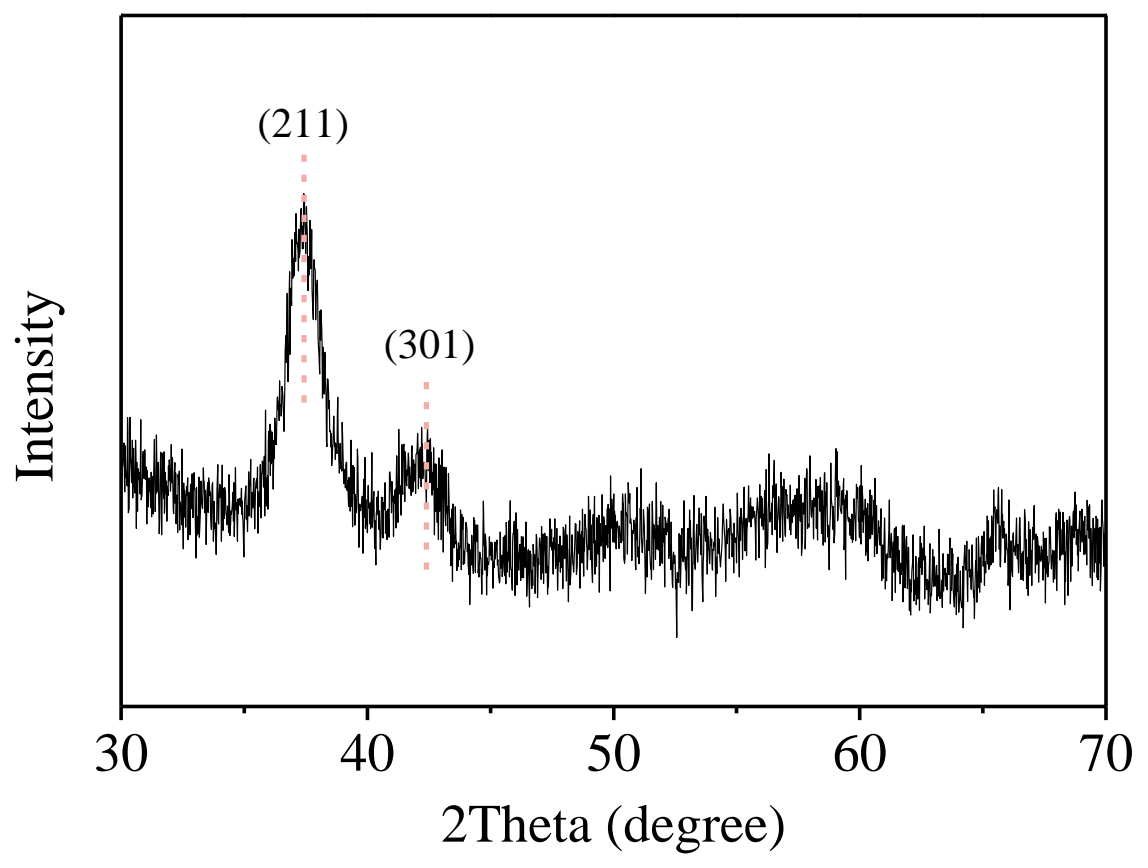

Figure S9. XRD pattern of the $\mathrm{NR}-\mathrm{MnO}_{2}$ catalyst after the $5^{\text {th }}$ run. 

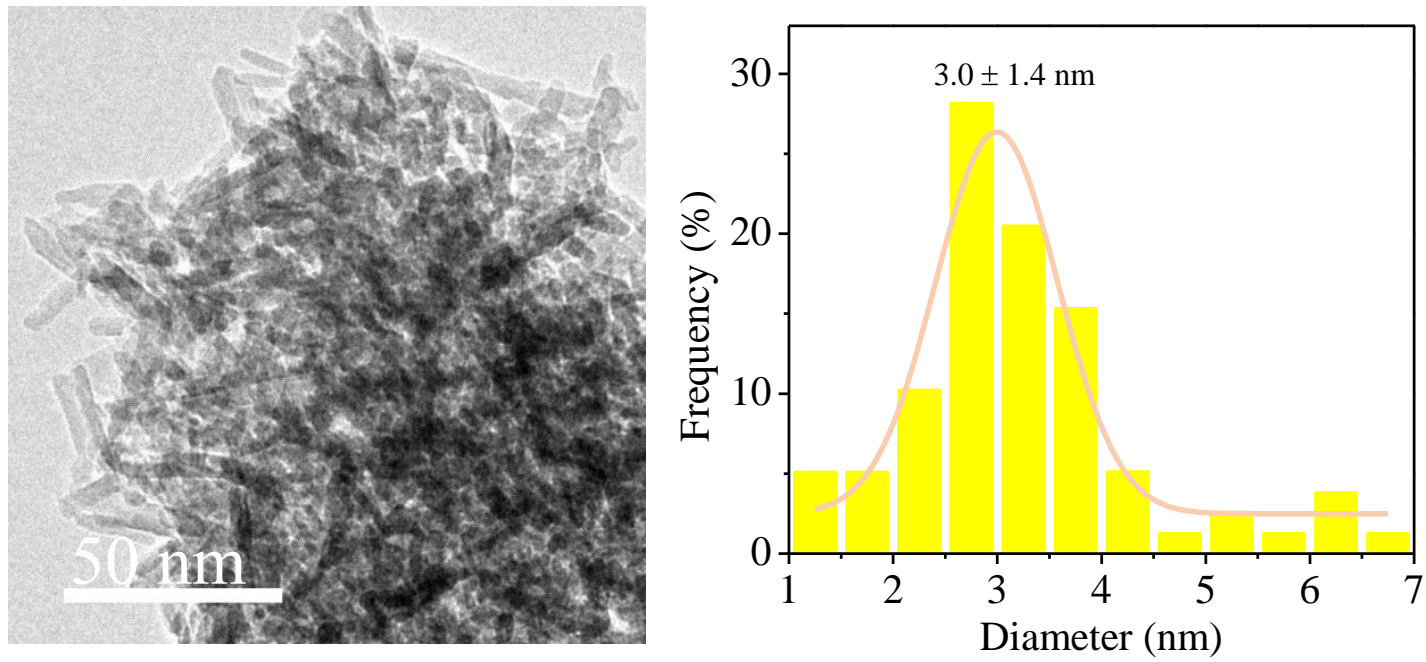

Figure S10. TEM image and the diameter distribution of the $\mathrm{NR}-\mathrm{MnO}_{2}$ catalyst after $5^{\text {th }}$ run. 


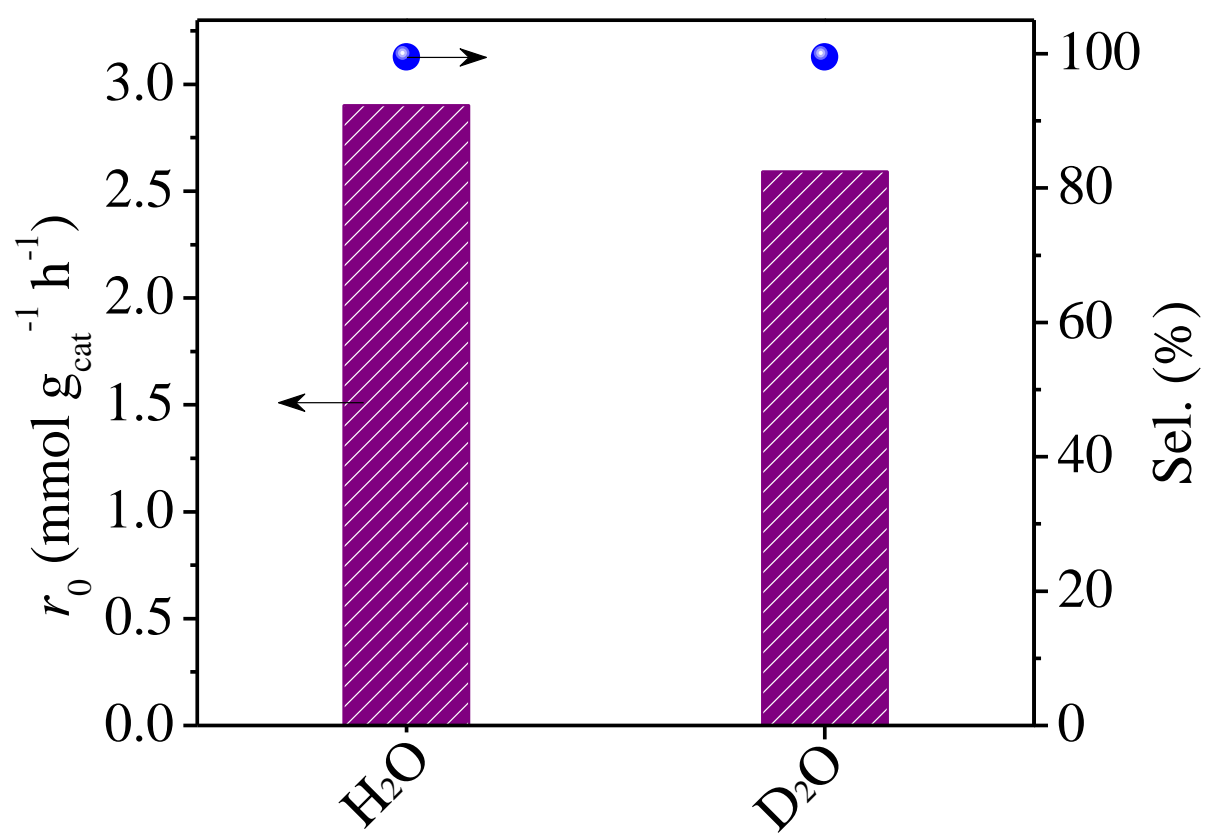

Figure S11. Average reaction rate $\left(r_{0}\right)$ and benzamide selectivity in the hydration of benzonitrile over $\mathrm{NR}-\mathrm{MnO}_{2}$ catalyst using $\mathrm{H}_{2} \mathrm{O}$ and $\mathrm{D}_{2} \mathrm{O}$. Reaction conditions: 0.4 mmol of substrate, $4 \mathrm{~mL}$ of $t$-amyl alcohol, $100 \mu \mathrm{L}$ of $\mathrm{H}_{2} \mathrm{O}$ or $\mathrm{D}_{2} \mathrm{O}, 100 \mathrm{mg}$ of catalyst, $75^{\circ} \mathrm{C}, 15 \mathrm{~min}$. 

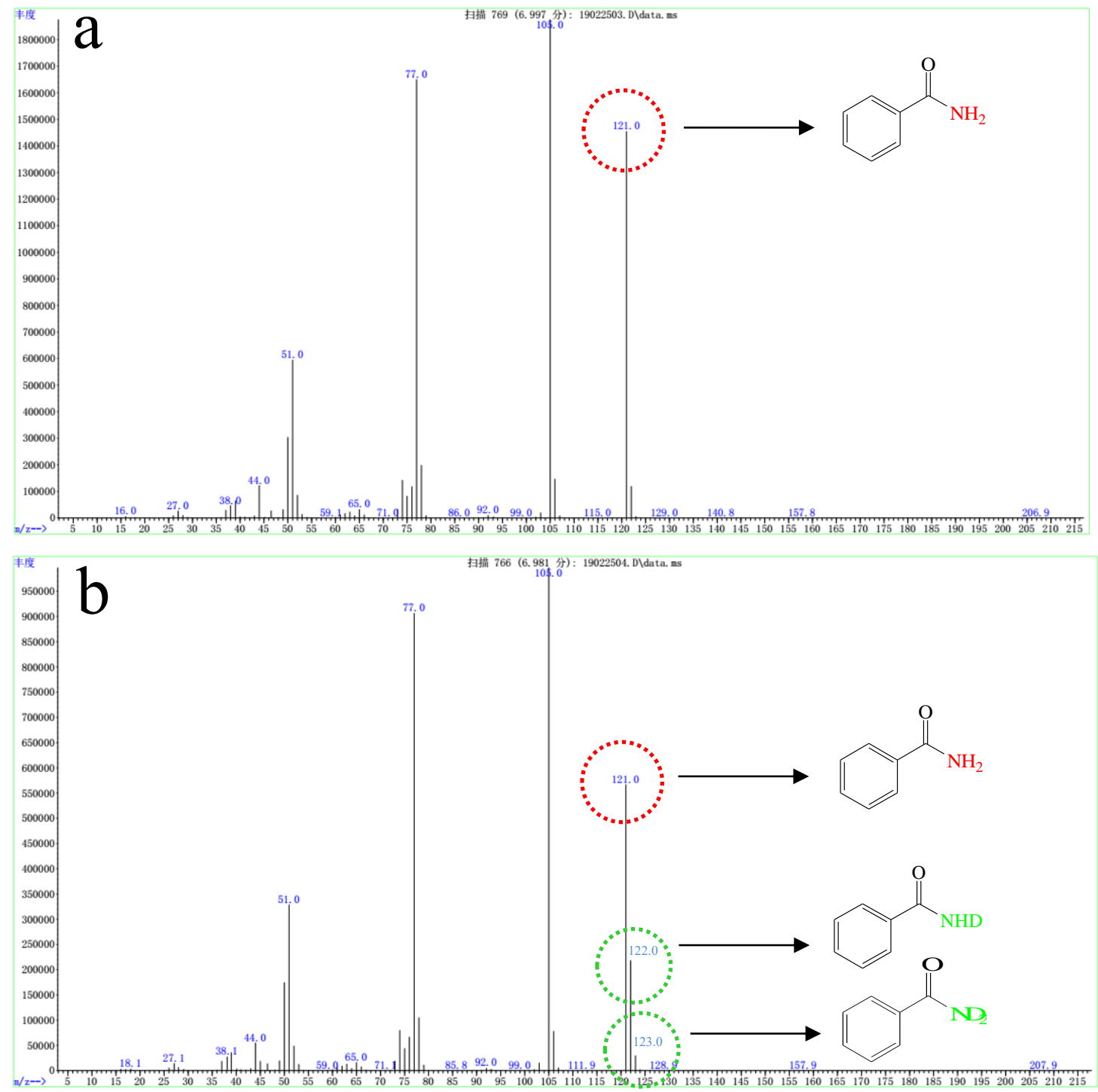

Figure S12. GC-MS spectra of the D-labeling experiment in benzonitrile hydration over NR-MnO ${ }_{2}$ catalyst. Reaction conditions: $0.4 \mathrm{mmol}$ of substrate, $4 \mathrm{~mL}$ of $t$-amyl alcohol, $100 \mathrm{mg}$ of catalyst, $75^{\circ} \mathrm{C}, 1.5 \mathrm{~h}, 100 \mu \mathrm{L}$ of (a) $\mathrm{H}_{2} \mathrm{O}$ and (b) $\mathrm{D}_{2} \mathrm{O}$. 


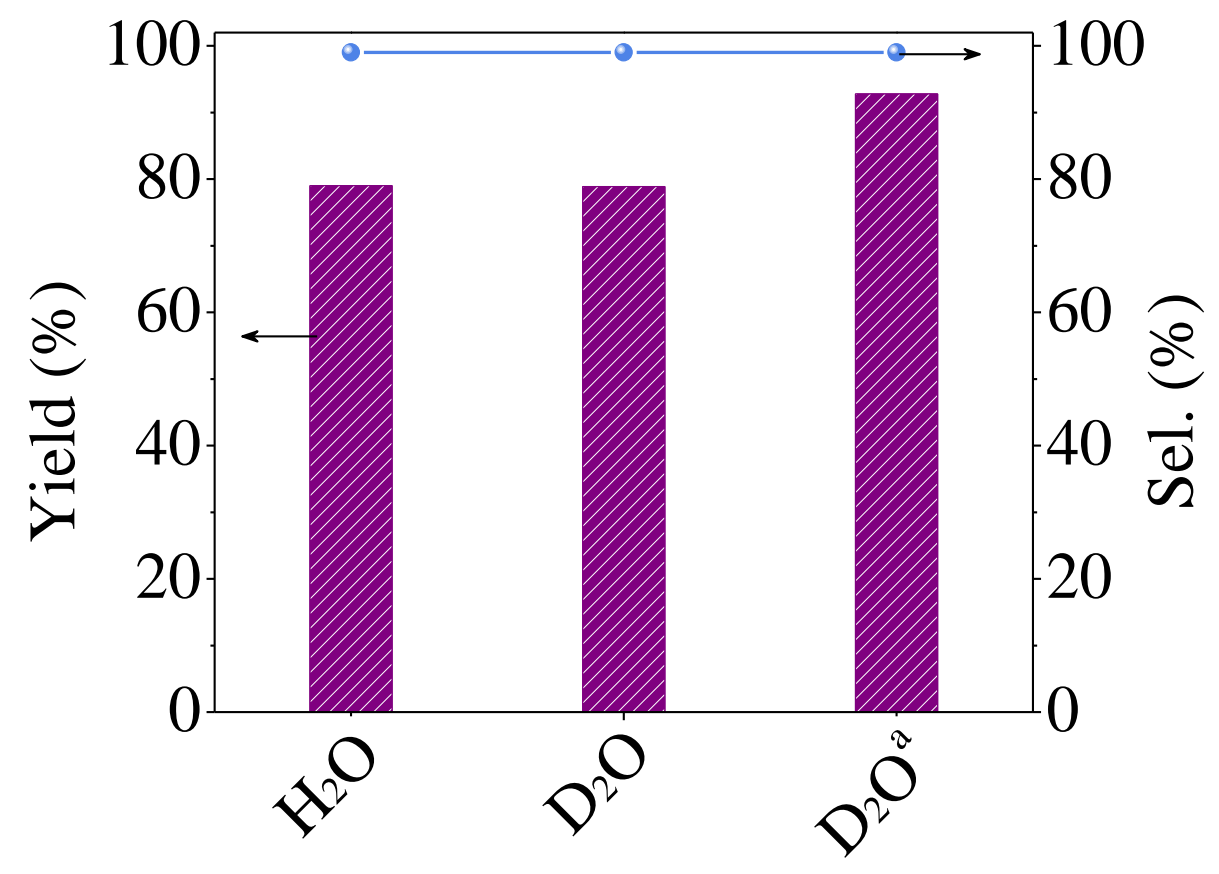

Figure S13. The yields and selectivities of benzamide in the benzonitrile hydration with $\mathrm{H}_{2} \mathrm{O}$ and $\mathrm{D}_{2} \mathrm{O}$. Reaction conditions: $0.4 \mathrm{mmol}$ of substrate, $4 \mathrm{~mL}$ of $t$-amyl alcohol, 100 $\mu \mathrm{L}$ of $\mathrm{H}_{2} \mathrm{O}$ or $\mathrm{D}_{2} \mathrm{O}, 100 \mathrm{mg}$ of catalyst, $75^{\circ} \mathrm{C}, 1.5 \mathrm{~h} .{ }^{a} 100{ }^{\circ} \mathrm{C}$. 


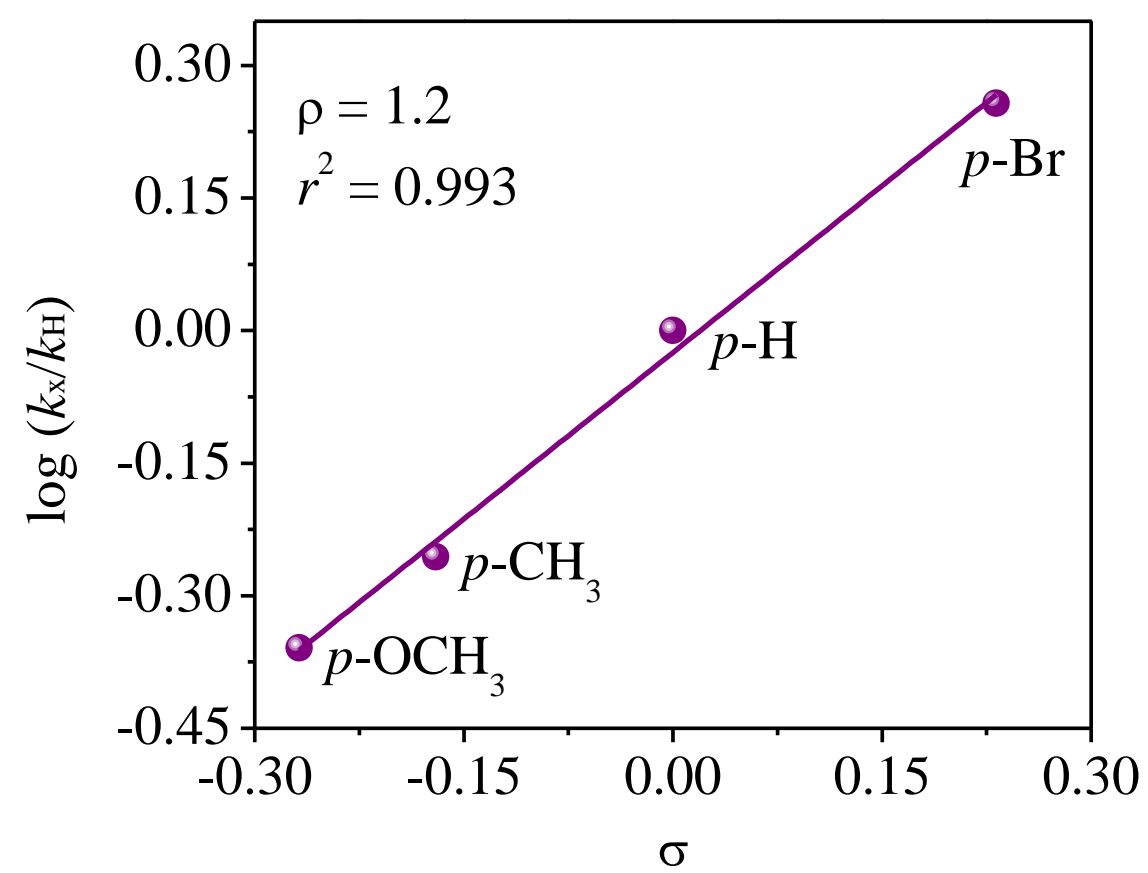

Figure S14. Hammett plots for the hydration of different $p$-substituted benzonitriles over the NR- $\mathrm{MnO}_{2}$ catalyst. Reaction conditions: $0.4 \mathrm{mmol}$ of substrate, $4 \mathrm{~mL}$ of $t$-amyl alcohol, $100 \mu \mathrm{L}$ of $\mathrm{H}_{2} \mathrm{O}, 100 \mathrm{mg}$ of catalyst, $75^{\circ} \mathrm{C}$. 
Table S1. Catalytic performance of various homogeneous catalysts and $\mathrm{NR}-\mathrm{MnO}_{2}$ in hydation of benzontrile.<smiles>N#Cc1ccccc1</smiles>

\begin{tabular}{|c|c|c|c|c|c|c|c|}
\hline & Catalyst & Additive & $\begin{array}{l}\text { Temp. } \\
\left({ }^{\circ} \mathrm{C}\right)\end{array}$ & $\begin{array}{l}\text { Time } \\
\text { (h) }\end{array}$ & $\begin{array}{l}\text { Conv. } \\
(\%)\end{array}$ & $\begin{array}{l}\text { Sel. } \\
(\%)^{b}\end{array}$ & Ref. \\
\hline 1 & Soluble Pd NPs & $\mathrm{Cu}(\mathrm{acac})_{2}$ & 100.0 & 160.0 & 91.0 & 87.0 & 2 \\
\hline 2 & {$\left[\{\mathrm{Rh}(\mathrm{OMe})(\mathrm{cod})\}_{2}\right] / \mathrm{PCy}_{3}$} & $\mathrm{PCy}_{3}$ & 25.0 & 24.0 & 99.0 & 99.0 & 3 \\
\hline 3 & {$\left[\mathrm{RuCl}_{2}\left(\eta^{6}-\mathrm{C}_{6} \mathrm{H}_{6}\right)\left\{\mathrm{P}\left(\mathrm{NMe}_{2}\right)_{3}\right\}\right]$} & None & 100.0 & 3.0 & 99.0 & 99.0 & 4 \\
\hline 4 & TBA-SiW10 + Pd(OAc) $)_{2}$ & None & 90.0 & 9.0 & 99.0 & 99.0 & 5 \\
\hline 5 & $\mathrm{TBA}-\mathrm{SiW} 10+\mathrm{Mn}(\mathrm{OAc})_{2} \cdot 4 \mathrm{H}_{2} \mathrm{O}$ & None & 90.0 & 9.0 & $<1.0$ & - & 5 \\
\hline 6 & $\mathrm{TpRu}\left(\mathrm{PPh}_{3}\right)\left(\mathrm{NHCOCH}_{3}\right)\left(\mathrm{H}_{2} \mathrm{O}\right)$ & None & 120.0 & 72.0 & 8.8 & 99.0 & 6 \\
\hline 7 & $\left(\eta^{5}-\mathrm{C}_{9} \mathrm{H}_{7}\right) \mathrm{Ru}(\mathrm{dppm}) \mathrm{H}$ & None & 120.0 & 72.0 & 80.0 & 99.0 & 7 \\
\hline 8 & $\begin{array}{l}\left\{\left[\left(\mathrm{PCy}_{3}\right)(\mathrm{CO}) \mathrm{RuH}\right]_{4}\left(\mu_{4}-\mathrm{O}\right)\left(\mu_{3^{-}}\right.\right. \\
\left.\mathrm{OH})\left(\mu_{2}-\mathrm{OH}\right)\right\}\end{array}$ & None & $\begin{array}{c}80.0-9 \\
0.0\end{array}$ & 8.0 & 99.0 & 99.0 & 8 \\
\hline 9 & $\begin{array}{l}{\left[\mathrm{RuCl}_{2}\left\{\kappa^{1}(P)-4-\right.\right.} \\
\left.\left.\mathrm{Ph}_{2} \mathrm{PC}_{6} \mathrm{H}_{4} \mathrm{CH}_{2} \mathrm{NHR}\right\}\left(\eta^{6}-\mathrm{Bu}\right)\right]\end{array}$ & None & 100.0 & 24.0 & 97.0 & 99.0 & 9 \\
\hline 10 & $\begin{array}{l}\text { cis }-\mathrm{Ru}(\mathrm{acac})_{2}\left(\mathrm{PPh}_{2} \mathrm{Py}\right)_{2} \\
(0.024 \mathrm{~mol} \% \text { of cat. })\end{array}$ & None & 180.0 & 10.0 & 38.0 & 99.0 & 10 \\
\hline 11 & $\mathrm{RuH}_{2}\left(\mathrm{PPh}_{3}\right)_{4}$ & $\begin{array}{l}\text { 2-dimethoxy } \\
\text { ethane }\end{array}$ & 120.0 & 24.0 & 92.0 & 99.0 & 11 \\
\hline 12 & {$\left[\mathrm{PtH}\left(\mathrm{PMe}_{2} \mathrm{OH}\right)\left(\mathrm{PMe}_{2} \mathrm{O}\right)_{2} \mathrm{H}\right]$} & None & 90.0 & 5.0 & 86.0 & 99.0 & 12 \\
\hline 13 & $\begin{array}{ll}{\left[\mathrm{RuCl}_{2}(p-\right.} & \text { Polyoxye } \\
\left.\text { cymene })\left(\mathrm{PPh}_{2} \mathrm{OEt}\right)\right] & \text { octyl cycl }\end{array}$ & $\begin{array}{l}\text { thylene( } 8) \text { iso } \\
\text { lohexyl ether }\end{array}$ & 100.0 & 8.0 & 97.0 & 99.0 & 13 \\
\hline 14 & $\mathrm{NR}-\mathrm{MnO}_{2}$ & None & 75.0 & 2.0 & 99.0 & 99.0 & $\begin{array}{l}\text { This } \\
\text { work }\end{array}$ \\
\hline
\end{tabular}


Table S2. Catalytic data of various catalysts in hydation of benzontrile. ${ }^{a}$

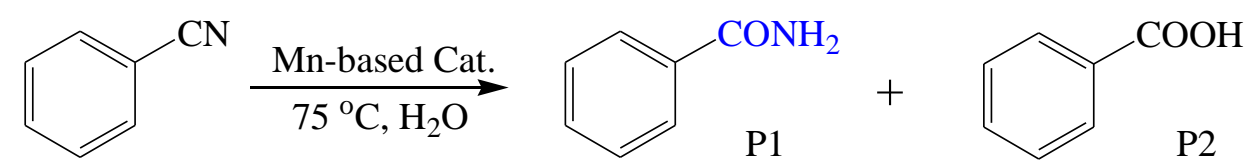

\begin{tabular}{|c|c|c|c|c|c|}
\hline & \multirow{2}{*}{ Catalyst } & \multirow{2}{*}{$\begin{array}{c}\text { Conv. } \\
(\%)\end{array}$} & \multicolumn{2}{|c|}{ Sel.(\%) } & \multirow[t]{2}{*}{ Balance $(\%)^{b}$} \\
\hline & & & P1 & $\mathrm{P} 2$ & \\
\hline 1 & Blank & $<0.1$ & n.d. & n.d. & $>99.5$ \\
\hline 2 & $\mathrm{~A}-\mathrm{MnO}_{2}$ & $<0.1$ & n.d. & n.d. & $>99.5$ \\
\hline 3 & $\mathrm{Mn}_{2} \mathrm{O}_{3}$ & $<0.1$ & n.d. & n.d. & $>99.5$ \\
\hline 4 & $\mathrm{Mn}_{3} \mathrm{O}_{4}$ & $<0.1$ & n.d. & n.d. & $>99.5$ \\
\hline 5 & $\mathrm{MnO}$ & $<0.1$ & n.d. & n.d. & $>99.5$ \\
\hline 6 & $\mathrm{Co}_{3} \mathrm{O}_{4}$ & $<0.1$ & n.d. & n.d. & $>99.5$ \\
\hline 7 & $\mathrm{Fe}_{3} \mathrm{O}_{4}$ & $<0.1$ & n.d. & n.d. & $>99.5$ \\
\hline 8 & $\mathrm{CeO}_{2}$ & $<0.1$ & n.d. & n.d. & $>99.5$ \\
\hline 9 & $\mathrm{MnSO}_{4}$ & $<0.1$ & n.d. & n.d. & $>99.5$ \\
\hline 10 & $\mathrm{KMnO}_{4}$ & $<0.1$ & n.d. & n.d. & $>99.5$ \\
\hline 11 & $\beta-\mathrm{MnO}_{2}$ & 20.9 & 92.3 & 6.8 & 99.1 \\
\hline 12 & $\mathrm{Pd} / \mathrm{C}$ & 22.2 & 96.5 & 3.1 & $>99.5$ \\
\hline 13 & $\mathrm{Ru} / \mathrm{C}$ & 23.5 & 95.1 & 3.9 & 99.0 \\
\hline 14 & $\mathrm{NR}-\mathrm{MnO}_{2}$ & $>99.5$ & $>99.5$ & $<0.1$ & $>99.5$ \\
\hline
\end{tabular}

${ }^{a}$ Reaction conditions: $0.4 \mathrm{mmol}$ of benzonitrile, $4 \mathrm{~mL}$ of $t$-amyl alcohol solvent, 100 $\mu \mathrm{L}$ of $\mathrm{H}_{2} \mathrm{O}, 100 \mathrm{mg}$ of catalyst, air, $75{ }^{\circ} \mathrm{C}, 2 \mathrm{~h} .{ }^{b}$ Carbon balance before and after the reaction. Conv., conversion. Sel., selectivity. n.d.: not detected. 
Table S3. Catalytic data of $\mathrm{NR}-\mathrm{MnO}_{2}$ in hydation of benzontrile at different temperatures. ${ }^{a}$

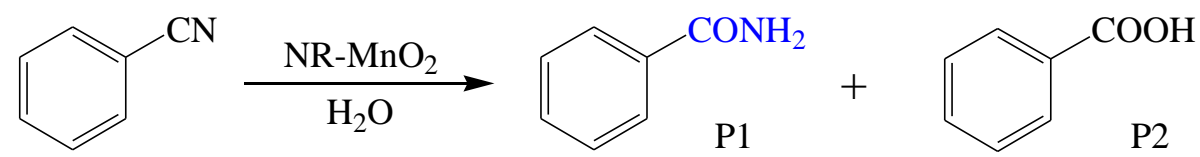

\begin{tabular}{cccccc}
\hline & \multirow{2}{*}{ Temp. $\left({ }^{\circ} \mathrm{C}\right)$} & Conv. & \multicolumn{2}{c}{ Sel. $(\%)$} & ${\text { Balance }(\%)^{b}}^{\text {B }}$ \\
\cline { 4 - 5 } & $(\%)$ & & P1 & P2 & \\
\hline 1 & 75 & $>99.5$ & $>99.5$ & n.d. & $>99.5$ \\
2 & 100 & $>99.5$ & $>99.5$ & n.d. & $>99.5$ \\
3 & 120 & $>99.5$ & $>99.5$ & n.d. & $>99.5$ \\
\hline
\end{tabular}

${ }^{a}$ Reaction conditions: $0.4 \mathrm{mmol}$ of benzonitrile, $4 \mathrm{~mL}$ of $t$-amyl alcohol solvent, 100 $\mu \mathrm{L}$ of $\mathrm{H}_{2} \mathrm{O}, 100 \mathrm{mg}$ of catalyst, air, $2 \mathrm{~h} .{ }^{b}$ Carbon balance before and after the reaction. Conv., conversion. Sel., selectivity. n.d.: not detected. 
Table S4. Catalytic data characterizing the $\mathrm{NR}-\mathrm{MnO}_{2}$ catalyst in hydation of benzontrile at different time. ${ }^{a}$

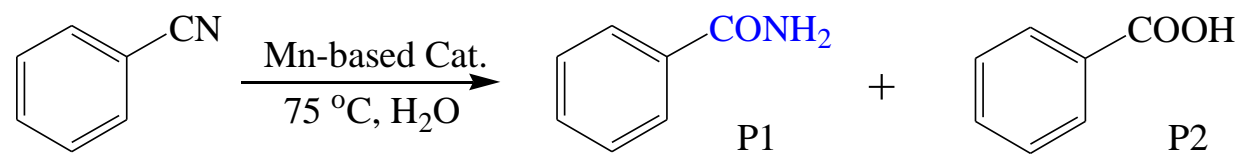

\begin{tabular}{cccccc}
\hline & \multirow{2}{*}{ Time (h) } & Conv. & \multicolumn{2}{c}{ Sel.(\%) } & ${\text { Balance }(\%)^{b}}^{\text {B }}$ \\
\cline { 5 - 6 } & $(\%)$ & \multicolumn{2}{c}{ P1 } & P2 & \\
\hline 1 & 0 & $<0.1$ & $<0.1$ & n.d. & $>99.5$ \\
2 & 0.25 & 20.6 & $>99.5$ & n.d. & $>99.5$ \\
3 & 0.5 & 40.0 & $>99.5$ & n.d. & $>99.5$ \\
4 & 1.5 & 79.0 & $>99.5$ & n.d. & $>99.5$ \\
5 & 2 & $>99.5$ & $>99.5$ & n.d. & $>99.5$ \\
6 & 3 & $>99.5$ & $>99.5$ & n.d. & $>99.5$ \\
7 & 4 & $>99.5$ & $>99.5$ & n.d. & $>99.5$ \\
8 & 12 & $>99.5$ & $>99.5$ & n.d. & $>99.5$ \\
\hline
\end{tabular}

${ }^{a}$ Reaction conditions: $0.4 \mathrm{mmol}$ of benzonitrile, $4 \mathrm{~mL}$ of $t$-amyl alcohol solvent, 100 $\mu \mathrm{L}$ of $\mathrm{H}_{2} \mathrm{O}, 100 \mathrm{mg}$ of catalyst, air, $75^{\circ} \mathrm{C} .{ }^{b}$ Carbon balance before and after the reaction. Conv., conversion. Sel., selectivity. n.d.: not detected. 
Table S5. Catalytic data in hydation of various ntriles over NR-MnO${ }_{2}{ }^{a}$

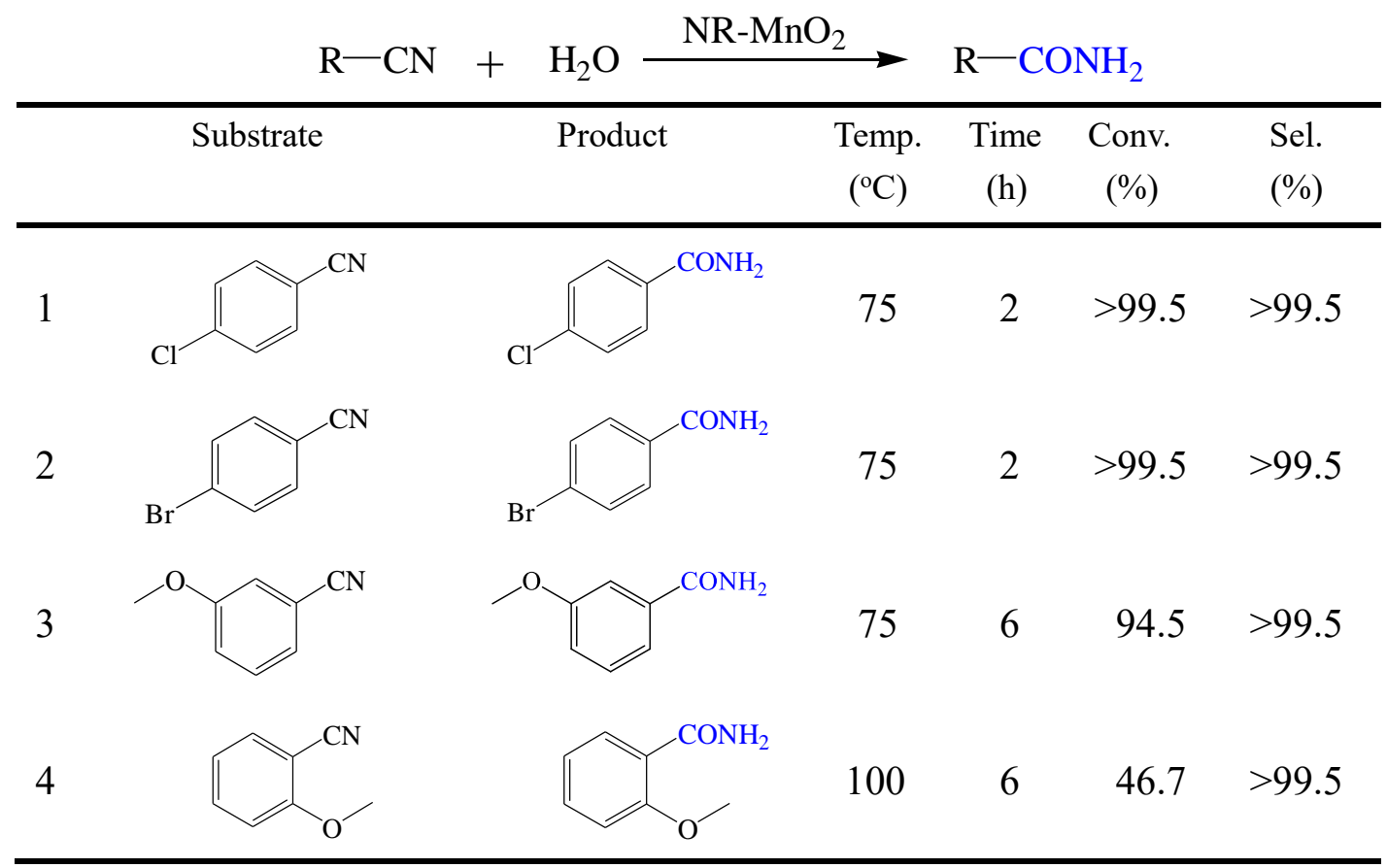

${ }^{a}$ Reaction conditions: $0.4 \mathrm{mmol}$ of substrate, $4 \mathrm{~mL}$ of $t$-amyl alcohol solvent, $100 \mu \mathrm{L}$ of $\mathrm{H}_{2} \mathrm{O}, 100 \mathrm{mg}$ of catalyst, 1 atm of air. Temp.: temperature; Conv., conversion; Sel., selectivity; n.d.: not detected. 


\section{REFERENCES}

(1) Deng, W.; Wang, Y.; Zhang, S.; Gupta, K. M.; Hülsey, M. J.; Asakura, H.; Liu, L.; Han, Y.; Karp, E. M.; Beckham, G. T.; Dyson, P. J.; Jiang, J.; Tanaka, T.; Wang, Y.; Yan, N. Catalytic amino acid production from biomass-derived intermediates. PNAS 2018, 115, 5093-5098.

(2) Ishizuka, A.; Nakazaki, Y.; Oshiki, T. The hydration of nitriles catalyzed by the combination of palladium nanoparticles and copper compounds. Chem. Lett. 2009, $38,360-361$.

(3) Goto, A.; Endo, K.; Saito, S. Rh-Catalyzed Hydration of organonitriles under ambient conditions. Angew. Chem. Int. Ed. 2008, 47, 3607-3609.

(4) García-Álvarez, R.; Díez, J.; Crochet, P.; Cadierno, V. Arene-ruthenium(II) complexes containing inexpensive tris(dimethylamino)phosphine: highly efficient catalysts for the selective hydration of nitriles into amides. Organometallics 2011, $30,5442-5451$.

(5) Hirano, T.; Uehara, K.; Kamata, K.; Mizuno, N. Palladium(II) containing $\gamma$-keggin silicodecatungstate that efficiently catalyzes hydration of nitriles. J. Am. Chem. Soc. 2012, 134, 6425-6433.

(6) Leung, C. W.; Zheng, W.; Zhou, Z.; Lin, Z.; Lau, C. P. Mechanism of catalytic hydration of nitriles with hydrotris(pyrazolyl)borato (Tp) ruthenium complexes. Organometallics 2008, 27, 4957-4969.

(7) Fung, W. K.; Huang, X.; Man, M. L.; Ng, S. M.; Hung, M. Y.; Lin, Z.; Lau, C. P. Dihydrogen-bond-promoted catalysis: catalytic hydration of nitriles with the 
indenylruthenium hydride complex $\left(\eta^{5}-\mathrm{C}_{9} \mathrm{H}_{7}\right) \mathrm{Ru}(\mathrm{dppm}) \mathrm{H} \quad(\mathrm{dppm} \quad=$ bis(diphenylphosphino)methane). J. Am. Chem. Soc. 2003, 125, 11539-11544.

(8) Yi, C. S.; Zeczycki, T. N.; Lindeman, S. V. Kinetic, spectroscopic, and X-ray crystallographic evidence for the cooperative mechanism of the hydration of nitriles catalyzed by a tetranuclear ruthenium- $\mu$-oxo- $\mu$-hydroxo complex. Organometallics 2008, 27, 2030-2035.

(9) García-Álvarez, R.; Díez, J.; Crochet, P.; Cadierno, V. Arene-ruthenium(II) complexes containing amino-phosphine ligands as catalysts for nitrile hydration reactions. Organometallics 2010, 29, 3955-3965.

(10)Oshiki, T.; Yamashita, H.; Sawada, K.; Utsunomiya, M.; Takahashi, K.; Takai, K. Dramatic rate acceleration by a diphenyl-2-pyridylphosphine ligand in the hydration of nitriles catalyzed by $\mathrm{Ru}(\mathrm{acac})_{2}$ complexes. Organometallics $\mathbf{2 0 0 5}, 24$, 6287-6290.

(11)Murahashi, S.-I.; Sasao, S.; Saito, E.; Naota, T. Ruthenium-catalyzed hydration of nitriles and transformation of .delta.-keto nitriles to ene-lactams. J. Org. Chem. 1992, $57,2521-2523$.

(12)Ghaffar, T.; Parkins, A. W. The catalytic hydration of nitriles to amides using a homogeneous platinum phosphinito catalyst. J. Mol. Catal. 2000, 160, 249-261.

(13)Cavarzan, A.; Scarso, A.; Strukul, G. Efficient nitrile hydration mediated by $\mathrm{Ru}^{\mathrm{II}}$ catalysts in micellar media. Green Chem. 2010, 12, 790-794. 